\title{
RADIATING SENSOR SELECTION FOR DISTRIBUTED BEAMFORMING IN WIRELESS SENSOR NETWORKS
}

\author{
Che-Wei Chang, Akshay Kothari, Ali Jafri, Dimitrios Koutsonikolas, Dimitrios Peroulis, Y. Charlie Hu \\ School of Electrical and Computer Engineering \\ Center for Wireless Systems and Applications and Birck Nanotechnology Center \\ Purdue University, West Lafayette, IN 47907
}

\begin{abstract}
Collaborative beamforming has already demonstrated its potential of significant power savings in distributed sensor networks. In collaborative beamforming, the antennas of the sensor nodes form a distributed antenna array in an effort to direct the radiated energy to the desired direction and thus increase the overall power efficiency of the network. Existing studies, however, have not addressed several major design issues: how to (1) optimally select a subset of radiating sensors for a given receiver to obtain optimal beamforming performance, (2) alternate among subsets of radiating sensors to prolong the lifetime of the sensors and robustness of network connection to the receiver, and (3) do so in the presence of synchronization and localization uncertainties. In this paper, we first show that the problem of selecting the subset of sensors that achieve optimal beamforming performance is NP-complete. We then propose a heuristic algorithm with complexity $\mathbf{O}(M \log M)$, where $M$ is the total number of distributed sensors, that simultaneously addresses the above three issues. In particular, we demonstrate its effectiveness in realistic scenarios with synchronization and localization errors. Further, we show that real-time grouping can be achieved even when thousands of sensors are spread over large distances of over 1000 wavelengths.
\end{abstract}

\section{INTRODUCTION}

Wireless sensor networks have been deployed for collecting critical data in various applications [1], [2], [3]. Such sensor networks can continuously record environment conditions without human intervention, and hence are ideal for monitoring forests, volcanoes, or swamps. Furthermore, they can be deployed in enemy territories in military applications, to track troop movement without risking the lives of scouts. Typical applications of wireless sensor networks have three common characteristics: (a) The sensors are deployed in areas without supporting facilities (such as wired network or energy sources). Consequently, these nodes must use energy judiciously to prolong the operational time of the networks. (b) Multiple sensors are deployed and form a multi-hop wireless network. However, the collected information must be transmitted to remote places (such as research facilities or military intelligence satellites) for analysis. (c) It is very difficult to regularly deploy these sensors because of the environmental constraints; As a result, the sensors' locations cannot be precisely known.

A major task of a wireless sensor network is to disseminate sensor readings back to a centralized host, called the sink node, for either real time monitoring (for example, environment monitoring) or/and reactive special event handling (for example, intrusion detection). Information dissemination to the sink node can be particularly challenging when the sink node is very far away from all informationgathering nodes. For example, the information-gathering nodes may be deployed in a hostile territory and the sink node may exist in a satellite or Unmanned Aerial Vehicle (UAV). In such cases the power required to transfer the acquired information to the sink node may be prohibitively high particularly for low-power nodes. Even if it is possible for a node to communicate directly to the sink node, it will deplete its battery very soon.

The idea of collaborative beamforming has been recently proposed as an efficient method for achieving the required information dissemination [4], [5], [6]. In collaborative beamforming, the nodes are forming a distributed antenna array in order to direct the transmitted energy to the desired direction and minimize radiation in all other directions. Due to reciprocity, collaborative beamforming is equally beneficial in the receive mode. The key conclusion of completed studies so far is that the achieved radiation patterns are on average acceptable. However, the results of a typical realization may be far from ideal [6]. In addition, no specific design methodology has been provided on how to form an efficient distributed array for a particular realization and in the presence of localization and synchronization uncertainties.

To address these important design issues of collaborative beamforming, our present work focuses on the following fundamental questions:

- Is there an optimal subset of nodes that forms a distributed antenna array with optimal performance? If yes, what is the computational cost of finding it?

- Provided that we usually prefer not to over- or underutilize a fixed subset of nodes, can we divide the available sensor nodes into a number of subsets such that each of them demonstrates reasonably good per- 


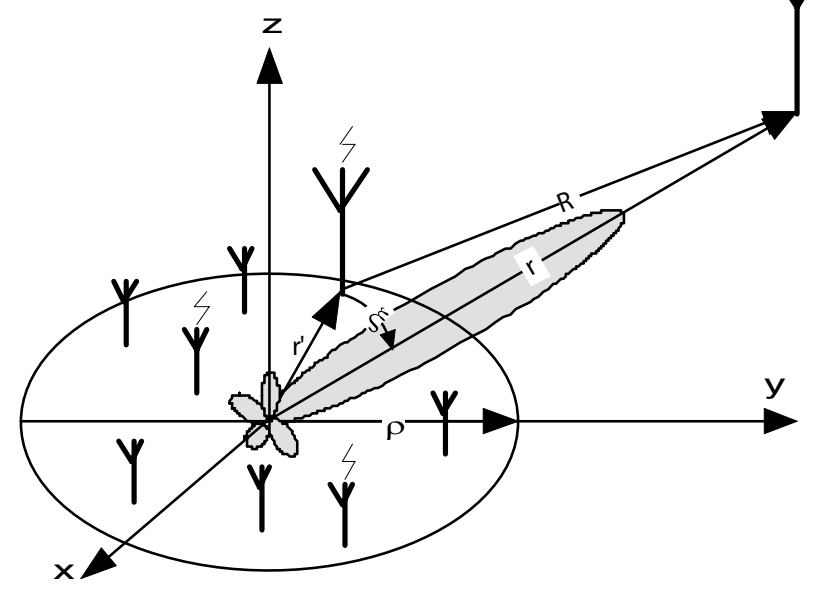

Fig. 1. Concept of distributed beamforming. The chosen nodes form a distributed antenna array and generate a directional radiation pattern towards the receiver.

formance and all are essentially equivalent to each other?

- How robust is this technique under localization and synchronization uncertainties?

This work addresses these questions by making the following assumptions: (a) the free-space propagation model is employed to simplify the analysis, (b) the sensors are assumed to be distributed on a plane, and (c) the MAC layer is already configured to allow collaborative beamforming. Our design methodology does consider, however, localization and synchronization uncertainties.

The remainder of this paper is organized as follows: Section II includes some background information and the overall problem definition (first question). Section III introduces our proposed heuristic algorithm and Section IV demonstrates its effectiveness on a wide variety of situations (second question). Section V discusses the impact of localization and synchronization uncertainties to the proposed method (third question).

\section{BACKGRound AND PROBlem Formulation}

In this section, we formally define the radiating sensor selection problem. Suppose there are $M$ sensors randomly deployed in an area with radius $\rho$, as shown in Fig. 1. The variables, $r, \theta$, and $\phi$, denote the radial distance, zenith, and azimuth, respectively. Primed and unprimed symbols refer to the sensor and the field points, respectively.

We assume that every sensor is equipped with an omnidirectional antenna that is linearly polarized along the z-axis, and each sensor has a random but slowly-variant initial phase, $\psi_{i}$, which corresponds to the initial time jitter through $\psi=\omega t_{j i t t e r}$. The frequency-domain electric field E with wavelength $\lambda$, radiated from the $i$-th sensor, at the point of interest is denoted as

$$
\mathbf{E}_{i}(r, \theta, \phi)=\exp \left(j\left(\psi_{i}-k R_{i}\right)\right) \hat{\mathbf{z}} .
$$

In (1), $\mathbf{E}_{i}$ has been normalized to unity, $k=\frac{2 \pi}{\lambda}$ is the wavenumber and $R_{i}=\left|\mathbf{r}-\mathbf{r}_{i}^{\prime}\right| \simeq|\mathbf{r}|-\left|\mathbf{r}_{i}^{\prime}\right| \cos \xi_{i}$ is the distance between sensor $i$ and the field point. From geometry, we have the angle $\xi_{i}$ between $\mathbf{r}$ and $\mathbf{r}_{i}^{\prime}$ equal to

$$
\cos \xi_{i}=\sin \theta \cos \left(\phi-\phi_{i}^{\prime}\right)
$$

We assume the field point is in the far-field region:

$$
r \gg 2 \frac{\rho^{2}}{\lambda} \quad(\text { for } \rho>\lambda),
$$

such that the field amplitude from each sensor is almost the same.

An antenna array is very effective in transmitting information over long distances because the radiated fields of its elements are being added coherently in the preferred direction of radiation [7]. For any given subset $p$ of sensors, the radiation intensity $U$ is given by

$$
U_{p}(\theta, \phi)=U_{0}(\theta, \phi)\left|A F_{p}(\theta, \phi)\right|^{2},
$$

where $U_{0}$ is the antenna element factor and $A F$ is the array factor:

$$
A F_{p}(\theta, \phi)=\sum_{q=1}^{m_{p}} \exp \left(j\left(\psi_{q}-k R_{q}\right)\right) .
$$

The directivity,

$$
D_{p}(\theta, \phi)=\frac{4 \pi U_{p}(\theta, \phi)}{\int_{0}^{2 \pi} \int_{0}^{\pi} U_{p}(\theta, \phi) \sin \theta d \theta d \phi},
$$

is defined as the ratio of the power radiated in a given direction to the average of the power radiated in all directions. The orientation of the radiation pattern is where the maximum directivity $D_{\max }$ located. Given the receiver orientation $\left(\theta_{R}, \phi_{R}\right)$, the radiation intensity and directivity at that orientation are given by $U_{p}\left(\theta_{R}, \phi_{R}\right)$ and $D_{p}\left(\theta_{R}, \phi_{R}\right)$, respectively.

Definition 1: (Radiating Sensor Selection Optimization Problem (RSSOP))

Assume a set of $M$ sensor nodes $S=\left\{s_{1}, s_{2}, s_{3}, \ldots, s_{n}\right\}$, each with coordinates $s_{i}=\left(r_{i}^{\prime}, \pi / 2, \phi_{i}^{\prime}\right)$, and a receiver $R$ in the far field with orientation $\left(\theta_{R}, \phi_{R}\right)$. The radiating sensor selection problem is to find a subset of sensor nodes $K_{p} \subset S$ along with their initial phase assignments such that (a) their distributed beamforming provides the maximal directivity towards the receiver $D_{\max }=D_{p}\left(\theta_{R}, \phi_{R}\right)$, (b) the chosen subset provides maximal directivity comparing to other possible subsets with the same number of sensor nodes, $D_{p}\left(\theta_{R}, \phi_{R}\right) \geq D_{q}\left(\theta_{R}, \phi_{R}\right)$. 


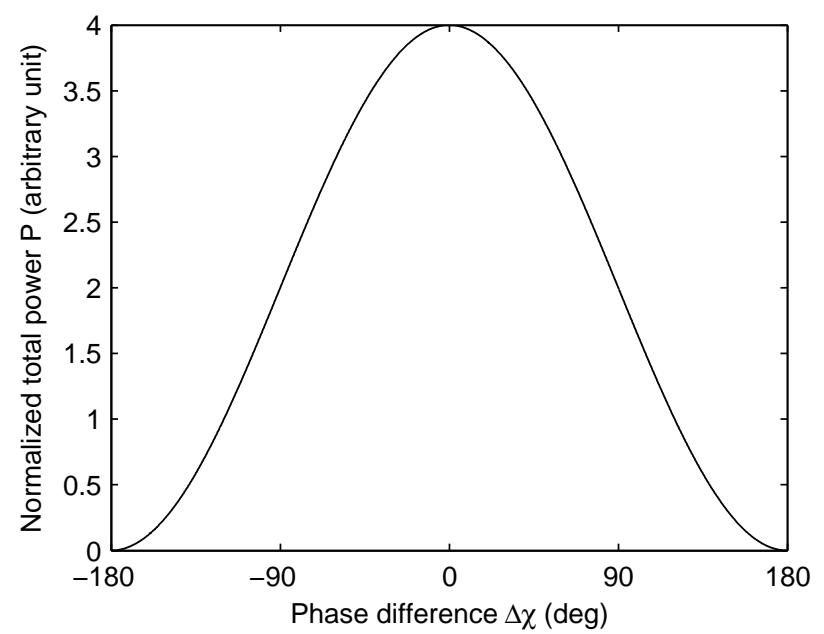

Fig. 2. Normalized power as function of relative phase difference for two sensor nodes. Maximum power is achieved for zero phase difference, but constructive interference is obvious for a wide range of angles.

\section{Proposed TeChNiQue: Phase Partition Method}

To the authors' best knowledge, it is not practical to solve the previously stated problem by explicitly computing the different combinations, because the problem complexity grows as $M$ !. Our proposed heuristic solution is discussed in the following.

\section{A. A Suboptimal Subset of Nodes}

To demonstrate the proposed technique, let's first consider two electromagnetic waves with different phases added together.

$$
\begin{aligned}
& E_{1}=\exp \left(j\left(-k R_{1}+\psi_{1}\right)\right)=\exp \left(j \chi_{1}\right), \\
& E_{2}=\exp \left(j\left(-k R_{2}+\psi_{2}\right)\right)=\exp \left(j \chi_{2}\right) .
\end{aligned}
$$

The total power is proportional to

$$
\begin{aligned}
P & =\left|E_{1}+E_{2}\right|^{2} \\
& =|1+\exp (j(\Delta \chi))|^{2},
\end{aligned}
$$

where

$$
\Delta \chi=\chi_{2}-\chi_{1}
$$

is the phase difference. As shown in Fig. 2, the smaller the phase difference, the higher the total power is.

While ideally we would require a zero phase difference between the waves, in practice, we will always have some phase error. As a result, instead of grouping nodes whose phases add perfectly, we choose to group the ones having approximately the same phase. This is the key idea of the method. For example, we could choose to form a group of sensors whose phases $\chi_{i}=\psi_{i}-k R_{i}$ are $0 \pm \Delta \chi$ deg., where $2 \Delta \chi$ is the phase span of the method and indicates our tolerance to phase matching. Using this technique, we can always find a suboptimal set of sensors whose radiation is added coherently but not necessarily optimally (depending on the phase span) along the receiver direction. Note that, once the receiver location is known, this suboptimal set of sensors can be very easily identified by simply checking their phases along the desired direction. Thus no expensive computations are needed.

Although it is always possible to integrate phase compensators [8], [9], [10] in the sensors, this increases the power consumption, noise and complexity of the sensors. For applications that need the simplest possible sensors, our proposed technique that does not require phase compensators may be preferable.

\section{B. Dividing the Available Sensor Nodes in Suboptimal Subsets}

It is interesting to note that the subset characterized by a phase of $0 \pm \Delta \chi$ deg. is not the only available one. Any subset that includes nodes with phases of $P \pm \Delta \chi$ deg, where $P$ is any given phase, will have the same performance. In other words, the received power depends only on the relative phase difference of the sensor nodes, note their absolute values.

Based on these ideas, we now explain how the Phase Partition Method separates all available sensors into subsets. Assuming that the phases are already acquired, first, each phase term $\chi_{i}$ is wrapped in the range $[0,2 \pi]$. Second, the $2 \pi$ span is equally separated to $N$ partitions, as shown in Fig. 3, so the phase span is $2 \Delta \chi=\frac{2 \pi}{N}$. Finally, sensors with phase in the same partition are grouped together. As a result, the average phase difference $\Delta \chi$ of each group is minimized during these processes. As long as $N>2$, each sensor in a group contributes the same sign of field; thus, no destructive interference occurs.

The optimal number of subsets depends on the application. Choosing smaller $N$ (more sensors in a subset) can yield higher $D_{\max }$. However, since the partition span is wider, some sensors may only contribute small field. Thus, the transmitted power is not as effective as grouping by larger $N$. The exact number of groups also depends on the expected phase uncertainties due to localization and synchronization errors. For example, it is unrealistic to group sensors whose phases differ less than $\pm 10 \mathrm{deg}$, when we expect a phase uncertainty of $\pm 60 \mathrm{deg}$. Additional details on the impact of phase uncertainty are given in Section IV.

For large $M$, the wrapped phases are expected to be uniformly distributed between $[0,2 \pi]$. Hence, each group will include approximately the same number of sensors and maximum directivity $D_{\max }$. As the partitions can be 


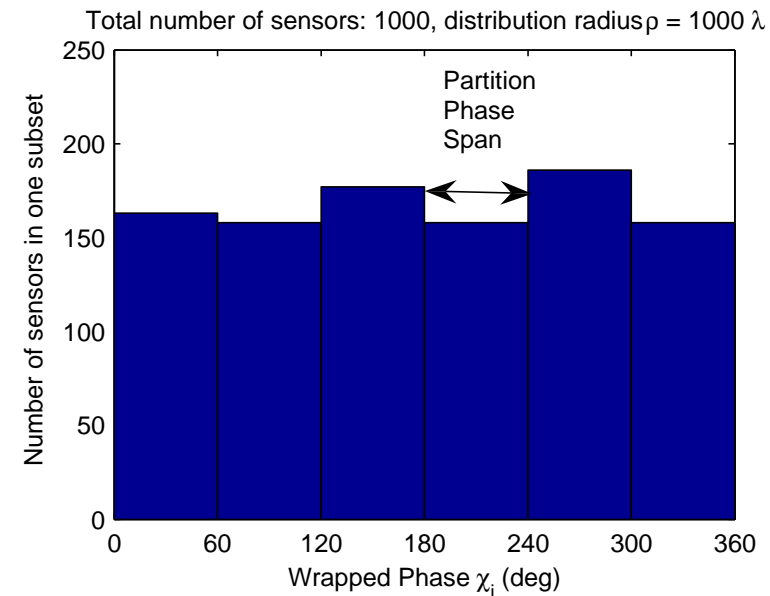

Fig. 3. Sensor distribution as a function of phase. The Phase Partition Method results in almost uniform distribution for large number of randomly distributed sensors.

circularly shifted, the grouping can be performed in an infinite number of ways. However, all solutions have the same $D_{\max }$. For small number of sensors, however, the distribution will not necessarily be uniform and further optimization may lead to additional benefits. Nevertheless, this is not an important case from a practical point of view since distributed beamforming has significant benefits in only large number of sensors.

To calculate the complexity of the Phase Partition Method, we consider its steps one by one: first, phase calculation and wrapping has a complexity of $\mathrm{O}(M)$; second, phase term sorting has a complexity of $\mathrm{O}(M \log M)$ by quick sort algorithm; third, grouping has a complexity $\mathrm{O}(N)$. All together, the complexity of the Phase Partition Method is $\mathrm{O}(M \log M)$.

\section{Simulation Results}

In order to assess the effectiveness of the proposed methodology, we considered a number of cases with various sensor node distributions. In each case we evaluated a) the maximum directivity $\left.D_{\max }, \mathrm{b}\right)$ the half-power $(3 \mathrm{~dB})$ beamwidth defined as the angle between the two directions in the main radiation lobe where the radiated power density is half of the maximum value, and c) the Side Lobe Level (SLL) defined as the ratio of the main lobe to the maximum side lobe. For each considered scenario, we ran a large number of cases and all resulted in very similar properties. In this section we present a summary of representative cases. The phase of each sensor node is assumed known until subsection D where we discuss the effects of phase uncertainties.

\section{A. Proof of concept}

Fig. 4 shows a case of 100 sensors randomly distributed in a disk of radius $\rho=1 \lambda$ (large radii are discussed later in this section). We assume that each sensor has a different initial phase $\psi_{i}$ (randomly assigned in the simulation code). The sensors are partitioned into six subset with a phase span of $60 \mathrm{deg}$. per subset. The (black) dots represent sensor nodes and the (red) circles indicate the chosen sensors in each subset. The receiver is located at $\phi_{R}=180^{\circ}$ on the plane of the sensors $\left(\theta_{R}=90^{\circ}\right)$ and marked as the blue line. The computed radiation pattern is normalized to $D_{\max }$ and shown in a logarithmic scale. The maximum directivity $D_{\max }$, half-power beamwidth, and the SLL are listed in the figure for each subset. As expected, each subset yields approximately the same performance. With an average of 16 sensors per subset, the system provides a $D_{\max }$ of 8.6, and a SLL of $6.9 \mathrm{~dB}$ on average. All subsets have maximum power pointed to the receiver. The differences between subsets could be further minimized by selecting a narrower phase width for the Phase Partition Method.

\section{B. Effect of Distribution Radius}

Depending on the frequency of operation, the sensors may be distributed over very large areas leading to sparse node distributions. This simulation studies the effect of the distribution radius $\rho$. The total number of sensors is kept constant at 1000 . The most noticeable effect is the change of pattern beamwidth. The half-power beamwidth decreases from 30 to less than $2 \mathrm{deg}$. as $\rho$ increases from 1 to $1000 \lambda$. The maximum directivities averaged over all 6 groups are also plotted in Fig. 5 as a function of distribution radius. The values saturate for $\rho>100 \lambda$. On the other hand, the SLL and $S T D\left(D_{\max }\right) / D_{\max }$ (STD: Standard Deviation), which are also plotted in the same figure, seem to be relatively independent of the distribution radius.

\section{Effect of number of sensors}

The effect of the number of the sensors is shown in Fig. 6 . The sensor number changes from 100 to 100,000 and the distribution radius is kept constant at $1000 \lambda$. Both $D_{\max }$ and SLL are linearly dependent on the number of sensors when plotted in a logarithmic scale. While the variation of $D_{\max }$ decreases as the number of the sensors increases, the standard deviation of the SLL shows a slower dependence.

\section{Effect of phase uncertainty}

In practice, the initial phase of each sensor node can be uncertain due to a) random shifting of its phased locked loop, oscillator and other circuit components, b) changes in temperature and other environment conditions, c) synchronization errors between sensors, d) localization errors, and 
Sensor Distribution

Total \# of sensors: 100

Distribution radius $=1 \lambda$

Subset 1,16 sensors
Radiation Pattern Sensor Distribution

Maximum Directivity $=9.5$ Total \# of sensors: 100

$3 \mathrm{~dB}$ beamwidth: 36 (deg) Distribution radius $=1 \lambda$

$\mathrm{SLL}=8.3(\mathrm{~dB})$

Subset 2, 18 sensors
Radiation Pattern

Maximum Directivity $=9.6$

$3 \mathrm{~dB}$ beamwidth: 32 (deg)

$\mathrm{SLL}=9.3(\mathrm{~dB})$

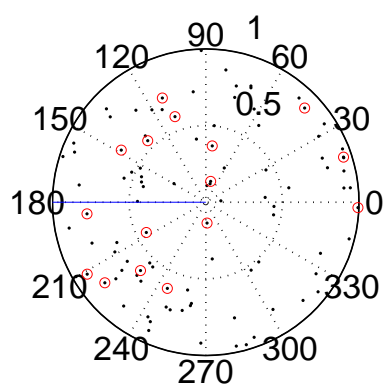

(a)

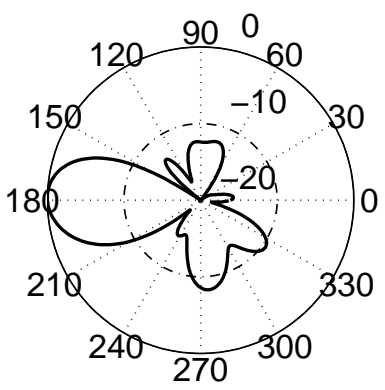

Sensor Distribution

Total \# of sensors: 100

Distribution radius $=1 \lambda$

Subset 3,17 sensors

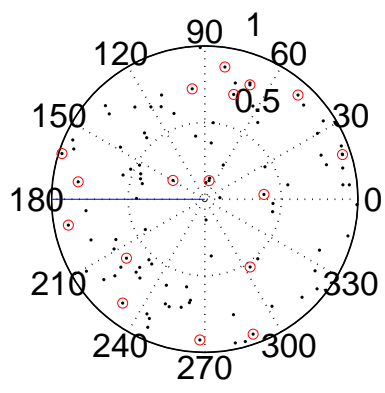

(c)

Radiation Pattern Sensor Distribution

Maximum Directivity $=9.0$ Total \# of sensors: 100

3dB beamwidth: 28 (deg) Distribution radius $=1 \lambda$

$\mathrm{SLL}=7.5(\mathrm{~dB})$
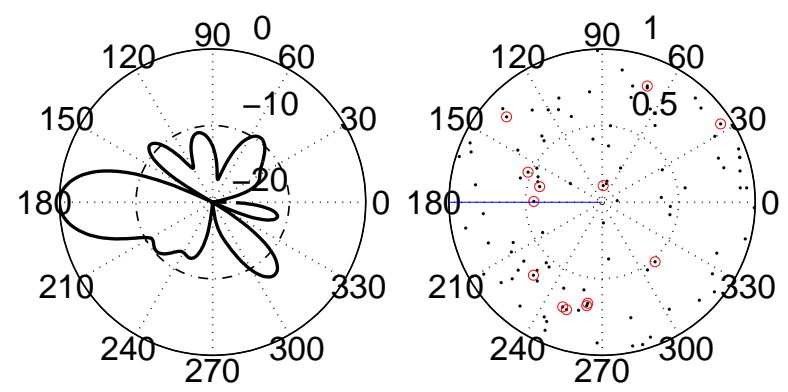

Sensor Distribution

Total \# of sensors: 100

Distribution radius $=1 \lambda$

Subset 5,24 sensors

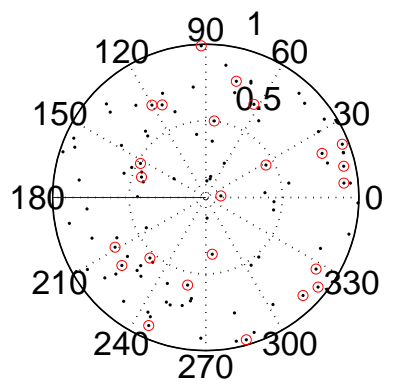

Radiation Pattern

Maximum Directivity $=9.9$

3dB beamwidth: 28 (deg)

$\mathrm{SLL}=6.7(\mathrm{~dB})$

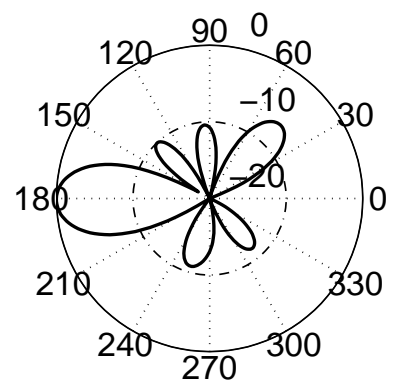

(e)
Subset 4,13 sensors

(d)
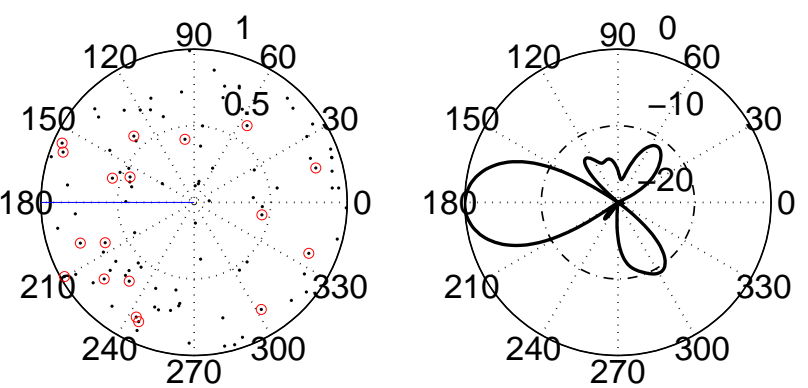

(b)

Radiation Pattern

Maximum Directivity $=5.6$

$3 \mathrm{~dB}$ beamwidth: 30 (deg)

$S L L=5.0(d B)$

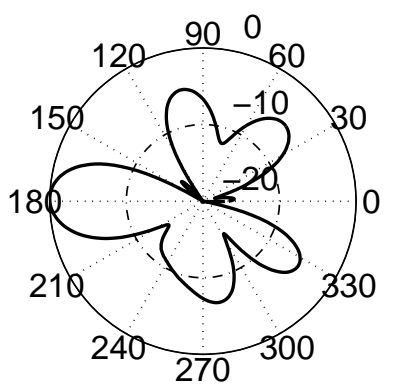

Sensor Distribution

Radiation Pattern

Maximum Directivity $=8.1$

$3 \mathrm{~dB}$ beamwidth: 28 (deg)

$\mathrm{SLL}=4.7(\mathrm{~dB})$

Fig. 4. Proof of concept. $M=100, \rho=1 \lambda . \bar{D}_{\max }=8.6, S T D\left(D_{\max }\right)=1.6 ; \overline{S L L}=6.9(d B), S T D(S L L)=1.8(d B)$. 


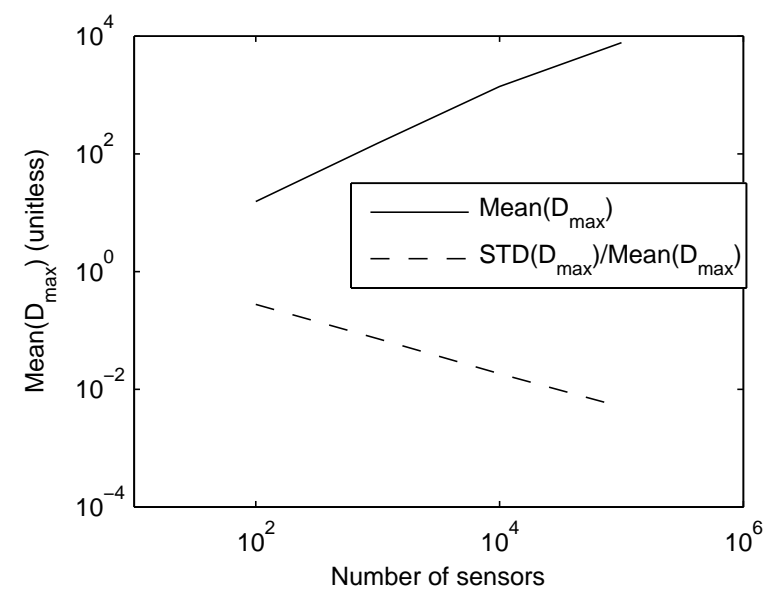

(a)

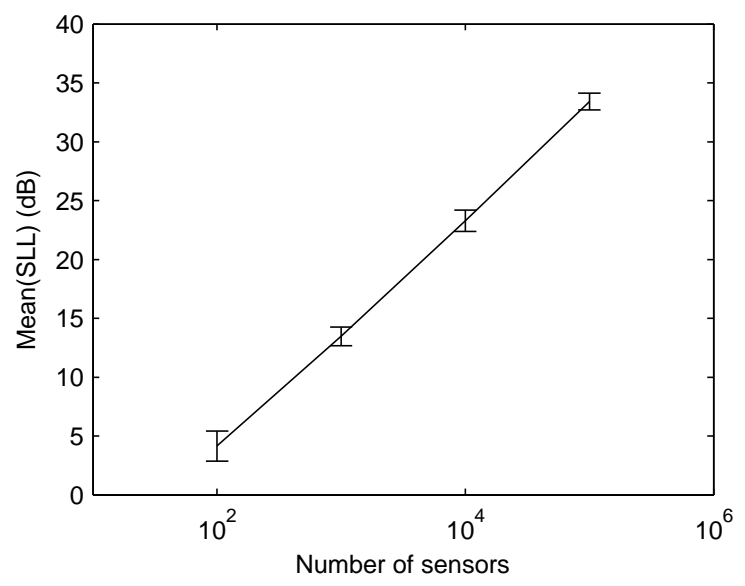

(b)

Fig. 6. Effect of number of sensors.

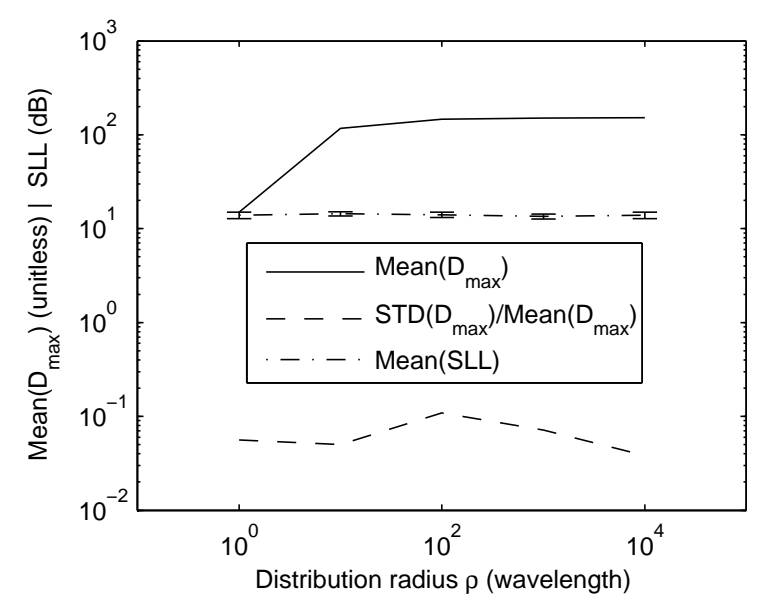

Fig. 5. Effect of distribution radius.

e) operating system limitations in each sensor. This simulation studies the effect of imperfect coherence due to these phase uncertainties. In the simulation, we first generate a set of sensor coordinates and initial phases. Next, we apply the Phase Partition Method and generate six groups of sensors with phase widths of $60 \mathrm{deg}$. Subsequently, we apply a random phase error in each sensor and we calculate the radiation pattern but without changing the partitions. This of course deteriorates the radiation pattern since each sensor may not belong to the correct group anymore. The random phase error is inserted in (5) as

$$
A F_{p}(\theta, \phi)=\sum_{q=1}^{m_{p}} \exp \left(j\left(\psi_{q}+\psi_{n q}-k R_{q}\right)\right) .
$$

The phase error is defined as

$$
\psi_{n q}=U(-W, W)
$$

where $U(-W, W)$ is an uniform random number generator in $[-W W], \mathrm{W}$ is the half width of the uniform random function. The result is shown in Fig. 7. The method is very robust and the radiation pattern preserves its main orientation even as the phase error goes up to $180 \mathrm{deg}$. For all six subsets, significant deviations start when the phase error approaches $300 \mathrm{deg}$.

This shows the robustness of the Phase Partition Method. As the phase span is $60 \mathrm{deg}$. in our simulation, adding a phase of 150 deg. can make a node change from constructive to destructive interference, which corresponds exactly to a $300 \mathrm{deg}$. full-width phase error. In rare and pathological cases we have observed that the main lobe changes to a different direction with a phase error smaller than complement value. This is because, with the additional phase error, some sensor subsets can form a better constructive interference at directions different than the main one. This is an example that Phase Partition Method may not guarantee $D_{\max }$ at the direction of receiver for $N=2$. However, this possibility decreases as $N$ increases. In our experience, $N=6$ is a safe number. All simulations have the radiation pattern pointing to the receiver.

\section{Discussion AND Future Work}

The proposed methodology presents a practical way for implementing collaborative beamforming in wireless sensor networks. While the algorithm does not necessarily result in the best possible performance, it does yield reasonably good results in most cases with low computational cost. In addition, it maintains its effectiveness in the presence of phase uncertainties due to a number of reasons including synchronization and localization issues. A number of interesting extensions can be considered towards implementing these ideas in practical collaborative sensing 


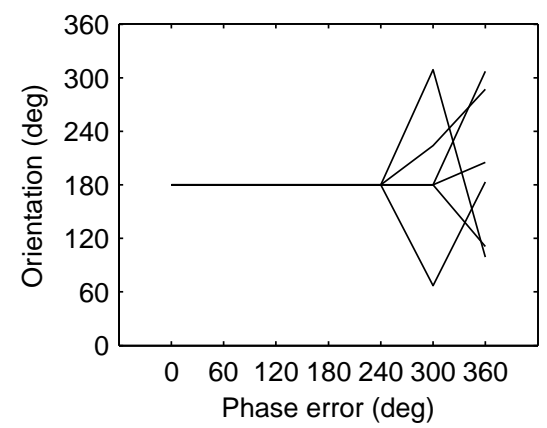

(a)

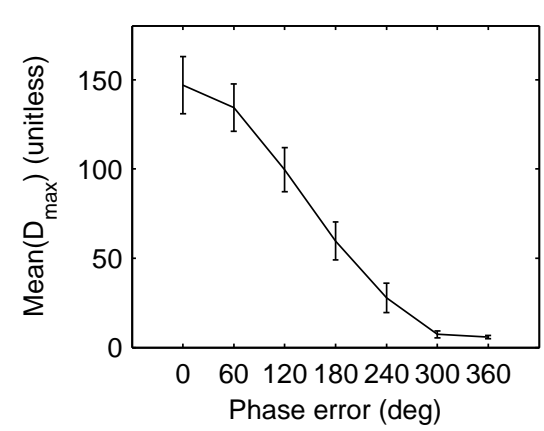

(b)

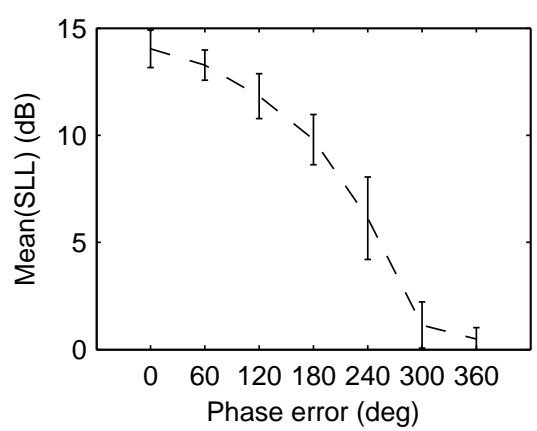

(c)

Fig. 7. Effect of phase uncertainty. $M=1000, \rho=100 \lambda$. All sensors are from subset 1 (159 sensors) with different phase noise $\psi_{n q}=U(-W, W)$. $U(-W, W)$ is an uniform random number generator. All groups have $3 \mathrm{~dB}$ beamwidth $<2$ (deg). The radiation pattern preserves its orientation when the phase error is smaller than the complement value of phase partition span.

networks. For example, it is likely that particular phase spans may yield optimal performance depending on the sensor node density per unit area. In addition, it would be interesting to consider the exact algorithm for grouping the sensor nodes in the desired subsets. While this could be accomplished with a few central nodes, it is possible that sensors far from the center of the constellation may improve the accuracy of the method. Furthermore, selecting an appropriate operation frequency will be key for the success of the method. Although achieving time synchronization for very high frequencies may prove particularly demanding [11], efficient antenna design at low frequencies is also very challenging [7]. Consequently, a compromise will be necessary to satisfy both requirements. Besides, it will be interesting to study the refinements necessary to the Phase Partition Method when more complex propagation models are considered and further optimization for longer battery life. Finally, some general questions for beamforming, such as how to initiate synchronous transmission, should the computation be done centrally or discretely? How does subsets communicate with each other? All these issues need to be carefully considered for obtaining functional sensor networks based on collaborative beamforming.

\section{CONCLUSION}

A new methodology for selecting the appropriate radiating elements in wireless sensor networks based on collaborative beamforming is presented. The proposed technique is based on a simple but effective algorithm for grouping all sensor nodes in subsets according to their initial potentially random phases and their locations relative to the desired receiver. A key advantage of this methodology is that results in several subsets each having essentially the same performance. Consequently, it allows the network designer to alternate between the different subsets thus extending the lifetime of the network. The paper presents the effectiveness of the proposed methodology for many different cases ranging from 100 to 100,000 sensors spreading over 1000 wavelengths. In addition, it demonstrates its robustness in the presence of phase uncertainties that result in phase errors of more than $180 \mathrm{deg}$.

\section{REFERENCES}

[1] Nirupama Bulusu and Sanjay Jha, Ed., Wireless Sensor Networks: A Systems Perspective. Artech House, 2005.

[2] A. Hac, Wireless Sensor Network Designs. John Wiley \& Sons, 2003.

[3] F. Zhao and L. Guibas, Wireless Sensor Networks : An Information Processing Approach. Morgan Kaufmann, 2004.

[4] C. Àlvarez, J. Díaz, J. Petit, J. Rolim, and M. Serna, "Efficient and reliable high level communication in randomly deployed wireless sensor networks," in MobiWac '04: Proceedings of the second international workshop on Mobility management \& wireless access protocols. New York, NY, USA: ACM Press, 2004, pp. 106-110.

[5] G. Barriac, R. Mudumbai, and U. Madhow, "Distributed beamforming for information transfer in sensor networks," in IPSN '04: Proceedings of the third international symposium on Information processing in sensor networks. New York, NY, USA: ACM Press, 2004, pp. 81-88.

[6] H. Ochiai, P. Mitran, V. Poor, and V. Tarokh, "Collaborative beamforming for distributed wireless ad hoc sensor networks," IEEE Transactions on Signal Processing, vol. 53, no. 11, pp. 4110 4124, November 2005.

[7] C. Balanis, Antenna Theory, Analysis and Design. John Wiley \& Sons, Inc., 1997.

[8] H. Hayashi and M. Mauraguchi, "An mmic active phase shifter using a variable resonant circuit [and mesfets]," Microwave Theory and Techniques, IEEE Transactions on, vol. 47, no. 10, pp. 20212026, Oct 1999.

[9] T. Hancock and G. Rebeiz, "A 12-ghz sige phase shifter with integrated lna," Microwave Theory and Techniques, IEEE Transactions on, vol. 53, no. 3, pp. 977-983, March 2005.

[10] H. Hashemi, X. Guan, and A. Hajimiri, "A fully integrated 24 ghz 8-path phased-array receiver in silicon," Solid-State Circuits Conference, 2004. Digest of Technical Papers. ISSCC. 2004 IEEE International, pp. 390-534 Vol.1, Feb. 2004.

[11] J. Elson, L. Girod, and D. Estrin, "Fine-Grained Network Time Synchronization using Reference Broadcasts," in Symposium on Operating Systems Design and Implementation, 2002, pp. 147-163. 